\title{
Bach Speaks: A Cortical "Language-Network" Serves the Processing of Music
}

\author{
Stefan Koelsch,*, ${ }^{\prime 1}$ Thomas C. Gunter,* D. Yves v. Cramon,* Stefan Zysset,* \\ Gabriele Lohmann,* and Angela D. Friederici* \\ *Max Planck Institute of Cognitive Neuroscience, Leipzig, Germany; and †Department of Neurology, \\ Harvard Medical School, 330 Brookline Avenue, Boston, Massachusetts 02215
}

Received October 30, 2000

The aim of the present study was the investigation of neural correlates of music processing with fMRI. Chord sequences were presented to the participants, infrequently containing unexpected musical events. These events activated the areas of Broca and Wernicke, the superior temporal sulcus, Heschl's gyrus, both planum polare and planum temporale, as well as the anterior superior insular cortices. Some of these brain structures have previously been shown to be involved in music processing, but the cortical network comprising all these structures has up to now been thought to be domain-specific for language processing. To what extent this network might also be activated by the processing of non-linguistic information has remained unknown. The present fMRI-data reveal that the human brain employs this neuronal network also for the processing of musical information, suggesting that the cortical network known to support language processing is less domain-specific than previously believed. $\odot 2002$ Elsevier Science (USA)

Key Words: brain; musi c; language; fMRI; Broca; Wernicke; superior temporal sulcus; superior temporal gyrus; insular cortex; modulation.

\section{INTRODUCTION}

In recent ERP-studies, brain responses reflecting the processing of musical chord-sequences were similar, although not identical, to brain activity elicited during the perception of language, in both musicians (Patel et al., 1998; Koelsch et al., in press) and nonmusicians (Koelsch et al., 2000a, 2001, 2002). While relatively early (around 180-350 ms) electrical brain responses to unexpected items in a structured sequence were often lateralized to the left when processing language (Friederici et al., 1993; Hahne and Friederici, 1999), they were often lateralized to the right when process-

\footnotetext{
${ }^{1}$ To whom correspondence and reprint requests should be addressed. Fax: 617-667-8695. E-mail: mail@stefan-koelsch.de.
}

ing music (Patel et al., 1998; Koelsch et al ., 2000a). The early brain responses (maximal around 200-350 ms) elicited by violations of musical regual rities were taken to reflect the processing of music-syntactic information (Patel et al., 1998; Koelsch et al., 2000a). Later brain responses (maximal around 500-550 ms) were hypothesized to reflect the processing of meaning information in music (Koelsch et al., 2000a).

In a recent MEG study, early brain responses hypothesized to reflect music-syntactic processing were localized in Broca's area and its right homologue (Koelsch et al., 2000b; Maess et al., 2001), areas known to be involved in syntactic language processing (J ust et al., 1996; Dapretto and Bookheimer, 1999; F riederici et al., 2000b; Meyer et al., 2000b). In that MEG study, however, the neural generators of later brain responses found with EEG could not be localized, raising the question which other areas in the brain might be involved in the processing of music, and whether there is further overlap of brain structures involved in the processing of music with brain structures known to be involved in the processing of language.

Recent brain imaging studies on language processing (with auditory stimulation) have shown that temporal and frontal areas of both hemisperes are involved in the processing of connected speech, with a preponderance of the left hemisphere for the on-line processing of syntactic features (Friederici et al., 2000a) and a preponderance of the right hemisphere for the on-line processing of prosody (for example, melody and metre of speech Meyer et al., 2000a).

The involvement of areas known to be involved in the processing of language has been shown for music processing, although only for melodies, i.e., one-part stimuli (e.g., Peretz et al., 1994), and only for single areas located either in temporal areas (e.g., Liegeois-Chauvel et al., 1998), or located in frontal areas (e.g., Zatorre et al., 1992). Interactions between frontal and temporal cortices (but excluding Wernicke's area) and frontal cortices have been reported for working memory of tonal pitch (Zatorre et al., 1994). Especially the supe- 


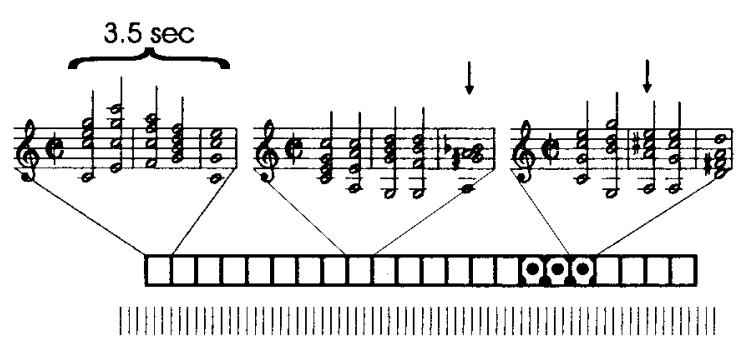

FIG. 1. Experimental paradigm. Top: examples of chord-sequences consisting of in-key chords only (left), terminated by a tonecluster (middle), and modulating (in the example from $C$ major to $D$ major, right). All sequence-types consisted of five chords and were presented in blocks comprising two to seven sequences of each type (middle row, in the example six in-key sequences are followed by four cluster sequences, etc.). All chords of these sequence-types were played on a piano. Each sequence had a duration of ca. $3.5 \mathrm{~s}$, sequences were presented one directly succeeding the other. Three functional images of nine slices each were continuously acquired per sequence (i.e., one image per bar, bottom row, each vertical line indicates one image). Subjects had to differentiate between in-key chords, clusters, and chords played by instruments deviating from piano (not depictured in this figure).

rior temporal gyrus (STG, Zatorre, 1984; Peretz, 1990; Platel et al., 1997; Liegeois-Chauvel et al., 1998; Zatorre et al., 1992) and the anterior portion of the right hemisphere, presumably including the right frontal lobe (Shapiro et al., 1981; Grossman et al., 1981) have been shown to be involved in music processing. However, up to now the network comprising the areas of both Broca and Wernicke has not been shown to be involved in music processing. This may partly be due to the fact that previous studies merely employed onepart stimuli (in contrast to Western tonal music, which is mostly multipart; for an imaging study investigating emotional responses to music with multi-part music see Blood et al., 1999).

In the present study, the experimental protocol was similar to previous EEG- and MEG-studies (Koelsch et al., 2000a, 2001, 2002; Maess et al., 2001): Chordsequences (i.e., multipart stimuli) were presented to nonmusicians, each sequence consisting of five chords (Fig. 1). One sequence directly followed the other, sounding like a musical piece. Most of the sequences were played by a piano and consisted of expected in-key chords only (left of Fig. 1). These sequences were composed in a way that a musical context was built up toward the end of each sequence, similar to the buildup of semantic context in a sentence (Krumhansl and Kessler, 1982; Koelsch et al., 2000a). During such a sequence, a strong expectancy for harmonically related chords is generated in the brains of listeners (Patel et al., 1998; Krumhansl and Kessler, 1982; Bharucha and Krumhansl, 1983; Bigand and Pineau, 1997; Tillmann et al., 2000; Koelsch et al., 2000a).

Infrequently, in some of the chord-sequences the tonal key was moved to another key (right of Fig. 1). Such a move is, in musical terms, denoted modulation and a very prominent stylistic feature of Western tonal music. Modulating chords were all perfectly consonant and harmonic, but they contained out-of-key notes (with respect to the preceding harmonic context) and were harmonically less related to the preceding harmonic context (e.g., in the sense of the circle of fifths; see Schönberg, 1969; Krumhansl and Kessler, 1982; Bharucha and Krumhansl, 1983; Patel et al., 1998). Since harmonically less related chords are perceived as more unexpected, modulating chords were perceived as more unexpected compared to the in-key chords (cf. Krumhansl and Kessler, 1982). Within a modulating chord sequence, a detection of a modulation could only be performed by the application of (implicit) knowledge about music-theoretical principles of harmonic distance and relatedness. These principles constitute the major-minor tonal system, i.e. the system of Western tonal music.

Moreover, some chord-sequences were not terminated by an expected in-key chord, but by a dissonant tone-cluster (middle of Fig. 1). In contrast to chords, these clusters had a highly dissonant, i.e. non-harmonic interval-structure. In addition, clusters contained out-of-key notes (like modulations), and were hence harmonically unrelated to the preceding harmonic context. That is, clusters violated musical expectancies of listeners to a higher degree than modulations and were, thus, more salient than modulations. Finally, in some sequences one or two in-key chords were played by an instrument other than piano (e.g., electric guitar, trumpet, organ). Participants were asked to detect sequences with clusters or deviant instruments, but instructed to respond behaviorally only to the deviant instruments (see Methods).

\section{METHODS}

Subjects. Ten nonmusicians participated in the experiment (20-29 years of age, 5 females). None of them had any special musical expertise or education, no subject had learned an instrument or had singing lessons. All subjects were right-handed and had normalhearing.

Stimuli. A pool of stimuli consisted of 34 different chord-sequences (each sequence consisted of five chords). The first chord was always the tonic of the following chord-sequence. Chords at the second position were tonic, dominant, mediant, submediant, subdominant. Chords at the third position were subdominant, subdominant with sixte ajoutée, dominant, dominant six-four chord, dominant seventh chord, and at the fourth position dominant seventh. Chords at the fifth position were tonic or cluster. Clusters consisted (with respect to the tonic) either of minor sixth, major sixth, and minor seventh, or of minor second, major second, and minor third. In modulating sequences, dominant chords at the second position were subdomi- 
nant of the new key, or mediants were the supertonic of the new key (both followed by a dominant chord of the new key at the third position, a dominant seventh chord of the new key at the fourth position, and the new tonic at the fifth position). Modulations thus stepped two fifths upwards and had a duration of three chords. Deviant instrument sequences consisted of inkey chords and contained one or two deviant instrumental sounds (General MIDI sounds from \#6 upwards; unsuitable sounds like \#36 [Slap-Base] were not used).

Sequences were composed with different melodic outlines (for example, beginning with the root, the third, or the fifth in the top-voice). Texture of chords followed the classical theory of harmony (Hindemith, 1940). Presentation time of chords 1- 4 was 585 ms, and of chord 5 was 1170 ms. All chords had the same decay of loudness. Chords were presented via earpipes and played under computerized control via MIDI on a synthesizer.

533 chord-sequences were randomly chosen in such a way that all sequence types (in-key, cluster-, modulating-, and deviant instrument-sequences) occurred in 153 blocks (distribution of blocks over functional sessions: 33/60/60). Each block of clusters, modulations, or deviant instruments was followed by a block of in-key chords. All chord-sequences were played in direct succession, there was no silent interval between chords, chord-sequences, or blocks, sounding like a musical piece. Blocks of clusters, modulations, and deviant instruments consisted of 2-4 (directly succeeding) sequences, in-key blocks consisted of at least 2, but not more than 7 sequences (see Fig. 1), resulting in 25 blocks with deviant instruments (containing 51 chordsequences), 25 blocks with clusters (containing 88 sequences), 25 with modulations (75 sequences), and 78 blocks with 319 in-key sequences (each functional session began and ended with a block of in-key chords).

Task. Participants were instructed to detect clusters and deviant instruments. They were asked to respond to deviant instruments by pressing a responsebutton. There were two buttons: the right button was to be pressed when no cluster had occurred since the last response, otherwise the left button had to be pressed. That is: no motor response was required when a cluster was detected, but the clusters were taskrelevant (since the detection of a cluster determined whether the left or right button had to be pressed for the next deviant instrument). Participants were not informed about the presence of modulations. The task enabled (a) to focus the attention of participants on both the timbral and the harmonic dimension of the stimuli, (b) to investigate the processing of harmonically deviant and task-relevant clusters without a motor response, and (c) to investigate the processing of harmonically deviant but task-irrelevant modulations.
MRI scanning procedure Scanning was performed on a 3-T Bruker Medspec 30/100 spectrometer. 9 axial slices (19.2 cm FOV, 64 by 64 matrix, $5 \mathrm{~mm}$ thickness, $2 \mathrm{~mm}$ spacing), parallel to the AC-PC plane (five slices above $A C-P C)$, using a single shot, gradient recalled EPI (TR $1170 \mathrm{~ms}$ ) were acquired. Three functional sessions of 1692 time points were run (blocks were introduced and ended with silence, thus 93 time points were not analyzed), each time point sampling over the nine slices. Prior to the functional recordings, anatomical slices were acquired. The anatomical slices had the same geometric orientation as the functional slices. In another session, a high-resolution T1-weighted fullbrain scan was obtained from each subject and geometrically rotated into a standard stereotactic coordinate system.

fMRI data analysis. fMRI-data were processed using the software package LIPSIA (Lohmann et al., in press). Functional data were corrected for slicetime acquisition differences using sinc-interpolation. Signal changes and baseline-drifts were removed by applying a temporal highpass filter to remove frequencies lower than $1 / 80 \mathrm{~Hz}$.

The anatomical slices were coregistered with the full-brain scan that resided in the stereotactic coordinate system and then transformed by linear scaling to a standard size. The transformation parameters obtained from this step were subsequently applied to the functional slices so that the functional slices were also registered into the stereotactic space.

The statistical evaluation was based on a leastsquares estimation using the general linear model for serially autocorrelated observations (see also Friston, 1994; Worsley and Friston, 1995; Aguirre et al., 1997; Zarahn et al., 1997). The design matrix was generated with a box-car function that included a response delay of $6 \mathrm{~s}$. The model equation, including the observation data, the design matrix and the error term, was convolved with a Gaussian kernel of dispersion of $4 \mathrm{~s}$ FWHM. The model includes an estimate of temporal autocorrelation that is used to estimate the effective degrees of freedom.

Four experimental conditions called in key, clusters, modulations, and deviant instruments were present where in key was used as a reference against which the other three conditions were contrasted. Thus, for each subject three contrast images were generated which represented the contrasts clusters vs in key, modulations vs in key, and deviant instruments vs in key.

As noted above, each individual functional dataset was aligned to a standard ster eotactic reference space, so that a group analysis based on the contrast images could be performed. The group analysis consisted of a one-sample $t$ test across the contrast images of all subjects that indicated whether the observed differences between conditions were significantly distinct 


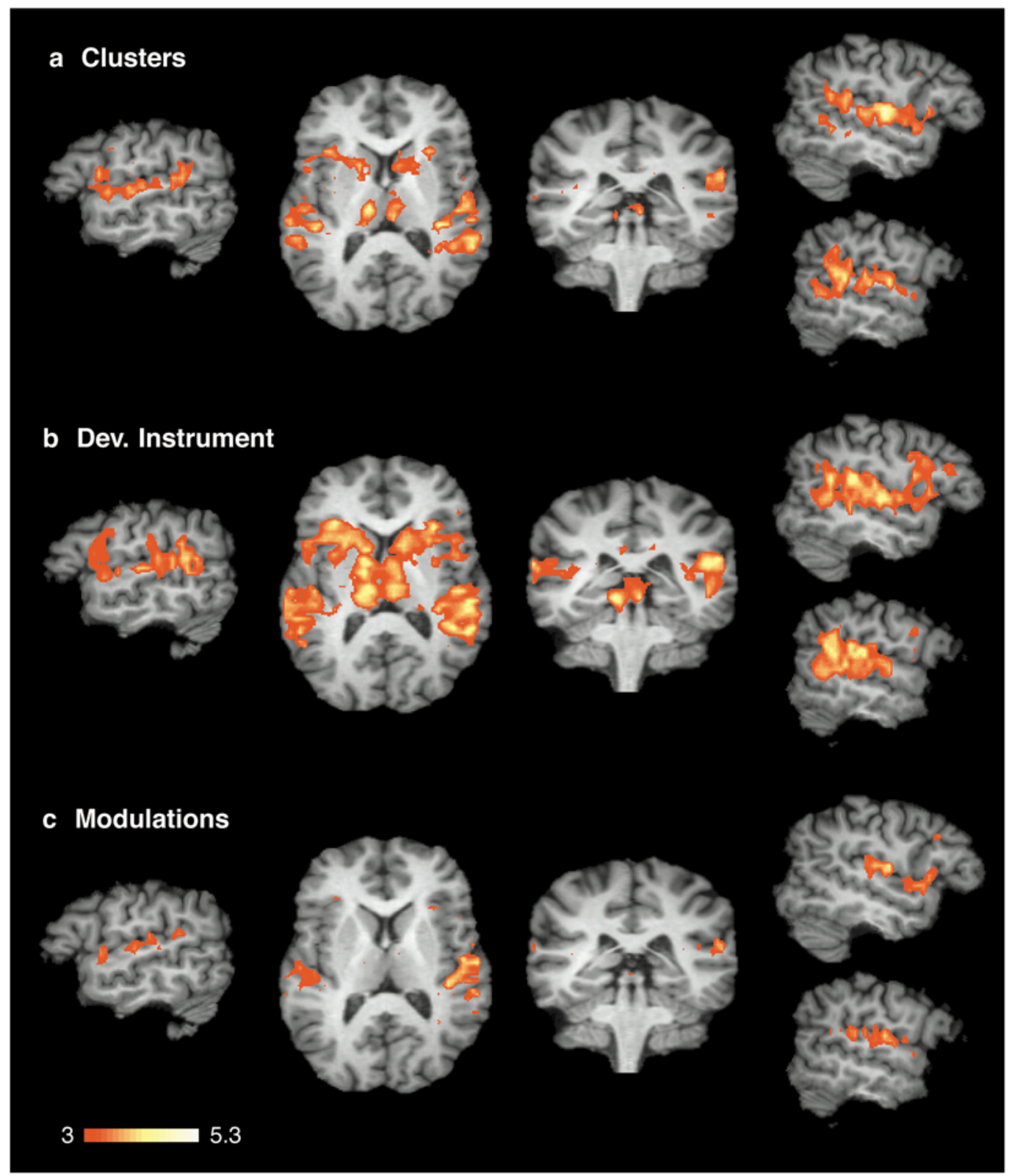

FIG. 2. Statistical z-maps of the contrasts clusters vs in-key (a), deviant instruments vs in-key (b), and modulations vs in-key (c), mapped onto an individual brain. Each panel shows views from left sagittal (left, Talairach coordinate $x=-52$ ), axial (middle-left, Talairach coordinate $z=14$ ), coronal (middle-right, Talairach coordinate $y=-33$ ), right mediosagittal (top right, Talairach coordinate $x=46$ ), and right laterosagittal (bottom right, Talairach coordinate $x=51$ ).

from zero (Holmes and Friston, 1998). This procedure was performed for all three contrasts resulting in three z maps shown in Fig. 2.

Table 1 lists local maxima of the $z$ maps. A pixel was defined to be a local maximum if its $z$ value exceeded 3.09 and if it was largest within a 5-mm radius. Local maxima residing in activation areas of size smaller than 150 cubic millimeters were not taken into account.

Areas that were found to be significantly activated were subjected to a further post hoc analysis. It was tested whether the activation strength in regions of interest (ROIs) differed between hemispheres and/or conditions (Bosch, 2000). In each hemisphere, six spheres were defined as ROIs (each sphere with a radius of $5 \mathrm{~mm}$ ). These ROIs were: BA 44, planum polare, anterior-superior insula, BA41, planum temporale (BA 22), and the area of Wernicke in the STG (BA $22 p)$.

The exact locations of the ROIs were established as follows: A new group z map was generated which resulted from contrasting the conditions clusters, modu- 
TABLE 1

Local Maxima of z-Maps, $x-, y-$, and z-Coordinates with Respect to Standard Stereotactic Space

(Talairach and Tournoux, 1988), Followed by Z Values

\begin{tabular}{|c|c|c|c|c|c|c|}
\hline \multirow{2}{*}{$\begin{array}{l}\text { Anatomical location } \\
\text { Left hemisphere }\end{array}$} & \multicolumn{2}{|c|}{ Clusters } & \multicolumn{2}{|c|}{ Deviant instruments } & \multicolumn{2}{|c|}{ Modulations } \\
\hline & & & & & & \\
\hline BA 44 & $-50,10,9$ & 3.5 & $-50,10,9$ & 3.8 & $(-50,9,5$ & 2.7) \\
\hline BA $44 / 6$ & $-44,7,23$ & 3.7 & $-47,10,22$ & 3.8 & & \\
\hline Insula & $-31,14,12$ & 4.2 & $-31,14,14$ & 4.3 & $-32,20,13$ & 3.1 \\
\hline STG/Planum pol. & $-47,4,-1$ & 4.4 & $-47,4,-1$ & 4.5 & $-50,2,0$ & 3.6 \\
\hline BA 41 & $-44,-22,13$ & 4.1 & $-44,-19,13$ & 4.5 & $-44,-20,12$ & 4.2 \\
\hline Planum temp. & $-48,-31,15$ & 4.1 & $-44,-31,16$ & 4.0 & $-47,-31,18$ & 3.4 \\
\hline \multirow[t]{2}{*}{ BA 22p } & $-53,-41,30$ & 4.3 & $-56,-37,28$ & 4.6 & $-56,-43,30$ & 4.5 \\
\hline & $-59,-40,18$ & 4.0 & $-53,-47,18$ & 4.1 & & \\
\hline Thalamus & $-10,-19,6$ & 4.7 & $-10,-16,8$ & 5.1 & $-7,-14,11$ & 3.0 \\
\hline Head of Caudate Nucl. & $-16,10,5$ & 4.0 & $-13,7,5$ & 4.3 & & \\
\hline \multicolumn{7}{|l|}{ Right hemisphere } \\
\hline BA 44 & $46,12,6$ & 3.6 & $46,9,6$ & 3.7 & $46,13,8$ & 3.5 \\
\hline BA $44 / 6$ & $40,15,25$ & 3.9 & $43,18,29$ & 3.8 & $43,14,28$ & 3.6 \\
\hline Insula & $28,23,12$ & 4.5 & $31,18,16$ & 4.0 & $29,23,9$ & 3.8 \\
\hline STG/Planum pol. & $49,2,2$ & 3.7 & $43,0,3$ & 4.1 & $49,2,2$ & 4.0 \\
\hline BA 41 & $46,-18,15$ & 4.4 & $46,-22,13$ & 4.2 & $46,-18,16$ & 3.7 \\
\hline Planum temp. & $55,-35,18$ & 4.1 & $46,-33,18$ & 4.7 & $52,-31,12$ & 3.8 \\
\hline \multirow[t]{2}{*}{ BA $22 p$} & $49,-44,23$ & 4.2 & $49,-44,23$ & 4.7 & $50,-44,24$ & 3.2 \\
\hline & $46,-58,17$ & 3.5 & $40,-55,17$ & 3.2 & $55,-45,14$ & 3.4 \\
\hline post. STS & $50,-44,17$ & 4.4 & $49,-43,17$ & 4.3 & & \\
\hline Thalamus & $8,-16,8$ & 4.0 & $5,-16,8$ & 5.0 & $3,-14,13$ & 3.4 \\
\hline Head of Caudate Nucl. & $14,14,0$ & 3.7 & $11,11,8$ & 4.1 & & \\
\hline
\end{tabular}

Note. A pixel was defined as local maximum whenever its $Z$ value exceeded 3.09 (corresponding to $P<0.001$ ), and if it was largest within a 5-mm radius. Local maxima residing in activation areas of size smaller than 150 cubic millimeters are not listed. In parenthesis: activation of left BA 44 by modulating sequences contrasted to in-key chords, thresholded with $z=2.58(P<0.005)$.

lations, and deviant against the in key -condition, so that all three experimental conditions of interest were represented in one z map. Each ROI was then centered at a local maximum of this $z$ map. Thus, the locations of the ROIs did not differ across conditions or subjects.

For all voxels of a ROI, a mean contrast was generated for each contrast and subject. These mean values subsequently entered a repeated measures ANOVA with factors condition, hemisphere and ROI. Since the ROIs analyzed in this post hoc ANOVA already were shown to be significantly activated, $\alpha$ was set to $\mathrm{P}=$ 0.05 with no further correction being necessary (Bosch, 2000).

\section{RESULTS}

The behavioral performance was on average above $95 \%$ correct responses, indicating that the given task could easily be mastered by the participants. The fMRI-data reveal that, contrasted to in-key chords, sequences terminated by a dissonant tone-cluster elicited a broad neuronal network (Table 1; Fig. 2a). This network comprised anterior-superior insular cortices and inferior fronto-lateral cortical areas (the lower part of BA 44, as well as its upper part, BA 44/6), the STG including the planum polare, Heschl's gyrus (BA 41), planum temporale (BA 42), the area of Wernicke in the
STG (BA 22p), and in the right hemisphere the posterior third of the upper bank of the superior temporal sulcus (STS, BA 22). This network (except the right STS) was activated in both hemispheres (an ANOVA with factor hemisphere and ROI did not reveal an effect of hemisphere; $P>0.55$ ).

Foci of maximal effects of chords played on deviant instruments (contrasted to sequences played on a piano) were fairly similar compared to effects of clusters (Table 1, Fig. 2b). In contrast to clusters, a lateralization of activations was statistically significant for deviant instruments: An ANOVA with factors hemisphere and ROI revealed an effect of hemisphere $(F(1,9)=6.41, P<0.05)$. Moreover, activations of deviant instruments were stronger than for clusters: An ANOVA with factor condition (clusters $x$ deviant instruments) and ROI revealed an effect of condition $(F(1,9)=253.36, P<0.0001)$.

A very similar network was activated by modulations, except that (with the threshold of $z=3.09$ ) no significant activations were found in the right STS and in the left pars opercularis (in contrast to both clusters and deviant instruments, cf. Table 1, Fig. 2c). However, with a threshold of $\mathrm{z}=2.58$ (corresponding to $\mathrm{P}<$ 0.005) a local maximum in the lower pars opercularis is also yielded for modulations. Similarly to clusters, an ANOVA with factor hemisphere and ROI did not reveal 
an effect of hemisphere ( $P>0.41)$. E ffects were weaker when elicited by modulations compared to clusters $(\mathrm{F}(1,9)=36.76, \mathrm{P}<0.0002)$.

In all three conditions, both the left and the right thalamus were activated, the activation being strongest when elicited by the deviant instruments, and weakest when elicited by the modulations. Additionally, a bilateral activation of the head of the caudate nucleaus (extending into the anterior putamen) was observed for the processing of deviant instruments and clusters, but not for modulations (cf. Table 1).

Note that the present contrasts between expected (in-key) and unexpected chords (clusters, modulations, and deviant instruments) cannot be due to the scanner noise because of the continuous stimulation, which was present during the presentation of both expected and unexpected chords. Moreover, it has been shown with both EEG and MEG that unexpected musical stimuli (similar to those used in the present) experiment elicit neural activity which is stronger compared to that elicited by expected chords (Koelsch et al., 2000a; Maess et al., 2001; Koelsch et al., 2001); hence, it is unlikely that the positive activations revealed in the present study are merely the result from a subtraction of deactivations.

\section{DISCUSSION}

A cortical network comprising numerous brain structures was activated by the processing of musical sequences. Several structures of this network have previously been shown to be involved in the processing of music: the right STG has in lesions studies been shown to be important for the processing of pitch (Zatorre, 1985; Samson and Zatorre, 1988; Zatorre and Samson, 1991). Patients with lesions in the the posterior STG bilaterally also show impairment of pitch perception (Liegeois-Chauvel et al., 1998), whereas the anterior STG has been shown to be involved in the processing of tonal and melodic processing as well as melodic representations (Zatorre, 1985; Samson and Zatorre, 1988; Zatorre et al., 1994; Platel et al., 1997; Peretz et al., 1994). The right frontal operculum (BA44) has been reported to be involved in working memory for pitch (Zatorre et al., 1994), and left BA44/6 has been reported to be involved in the processing of sequential sounds (Platel et al., 1997). The present data show that a cortical network comprising these structures in both hemispheres serves the processing of music in the (intact) human brain.

It is important to note that the structures activated in the present experiment are also well known to be involved in the processing of language, as revealed by studies with both auditory (Zatorre et al., 1992; Mummery et al., 1999; Meyer et al., 2000b; Pöppel, 1996; Friederici et al., 2000a; Binder et al., 1994; Schlosser et al., 1998; Bellin et al., 2000; Friederici, 1998) and vi- sual stimuli (J ust et al., 1996; Shaywitz et al., 1995; Mazoyer et al., 1993). Especially the areas of Broca and Wernicke have been shown in numerous lesion- and imaging-studies to be critically involved in the processing of language. The interdependence of the structures activated in the present study has so far only been observed for the processing of language, leading to the assumption that these structures are part of a language-specific neuronal network. The network activated in the present study appears to be very similar compared to the network that serves the understanding of language. This finding, especially in combination with the presented findings of previous studies on music processing, strongly suggests that the cortical language network is less domain-specific than previously believed.

The pars opercularis (BA 44, particularly its lower part), and the planum polare (anterior division of the STG), have been reported in several brain imaging studies to correlate with syntactic aspects during sentence comprehension (Friederici, 1998; Meyer et al., 2000b; Friederici et al., 2000a). With respect to (inferior) BA 44, the present results replicate findings of a recent MEG study (Maess et al., 2001; Koelsch et al., 2000b). In that study, a neuroanatomical correlate of music-syntactic processing has been localized within this area (note that the maximum of $\mathrm{fMRI}$ activation is located within a 5-mm radius of the dipole solution reported in the MEG study). The term musical syntax has been suggested to refer, at least partly, to the principles of harmonic distance and relatedness described by music theory: Within a sequence of chords, listeners expect the chords to be arranged harmonically closely related to each other (Maess et al., 2001; Krumhansl and Kessler, 1982; Bharucha and Krumhansl, 1983; Swain, 1997; Bharucha and Stoeckig, 1986; Koelsch et al., 2000a). Moreover, listeners expect within a sequence of in-key chords music-syntactically appropriate chord functions (Maess et al., 2001; Bigand and Pineau, 1997; Bigand et al., 1999). In the present study, both clusters and modulations were harmonically and functionally less related to the preceding chords of the chord-sequences (and did, thus, not fit into the tonal arrangement established by the preceding in-key chords). Hence, the processing of harmonic and functional relations (needed to decode the syntactic structure of the chord-sequences) was more difficult for clusters and modulations, possibly correlating with the activation of (inferior) BA 44. It might seem puzzling that deviant instruments also elicited areas of music-syntactic processing (since the chord-functions were harmonically appropriate), but the decoding of the syntactic structure of deviant-instrument sequences was more difficult because the different sounds presumably interfered with the harmonic analysis of the chord-functions. 
Wernicke's area is known to process the lexical-semantic aspects, i.e., meaning, of language (Friederici, 1998). Up to now, there is no clear consensus of how musical semantics might be defined (Swain, 1997; Raffmann, 1993; Tillmann et al., 1998; Platel et al., 1997; Koelsch et al ., 2000a). However, one could take to view that clusters, modulations, and deviant instruments belonged, within the contextual (and hence meaningful) harmonic environment, to different semantic categories compared to the in-key chords played on a piano. Notably, dissonances, modulations, and changes in instrumental timbre have a musical meaning which is utilized by composers as a means of expression (Swain, 1997). The processing of cluster, modulating, and deviant-instrument sequences might have activated Wernicke's area in an attempt to find a meaning of these acoustic events consistent with the previous musical passage.

The regions between the planum temporale and the upper bank of the posterior STS (bilaterally) have in a recent $\mathrm{fMRI}$ study been hypothesised to be voice-selective areas in the human brain (Bellin et al., 2000). In that study, these regions showed greater neuronal activity when subjects listened passively to vocal sounds, whether speech or nonspeech. In the present study these regions were in the right hemisphere activated by nonvocal stimuli, which might challenge, at least in the right hemisphere, a hypothesis of a strict voiceselectivity of this region.

Surprisingly, activations were observed for the processing of modulations, although modulations were not salient deviants and could only be differentiated from in-key chord sequences by the application of (implicit) knowledge about the rules of harmony (which constitute the major-minor tonal system). Since participants were nonmusicians, the present results hence support the hypothesis that the human brain has a general ability for a highly differentiated processing of music (see also Koelsch et al., 2000a; 2001; Maess et al., 2001), the acquisition of musical regularities most presumably being culture-specific.

With respect of our recent MEG study (Maess et al., 2001) it is interesting to note that brain responses with a latency of around 500 ms observed with EEG (e.g., Koelsch et al., 2000a) could not be localized with MEG (these responses are presumably generated by a number of overlapping neural sources, reducing the reliability of a source localization with MEG). The later electric brain responses were taken to reflect harmonic integration processes, in reminiscence of semantic integration processes during language perception. According to the results of the present experiment, which employed an experimental protocol very similar to the mentioned EEG experiments (Koelsch et al., 2000a), it seems highly probable that the later brain responses measured with EEG were generated in supratemporal lobe structures.
The finding that, in contrast to previous imaging studies on music processing, the described network was activated in the present study might be explained by the nature of the employed stimuli. In contrast to previous imaging studies, musical stimuli of the present study were multi-part, hence containing considerably more harmonic information compared to melodic stimuli: Although a single melody also has an implicit harmonic structure, the amount of harmonic information for each melody tone is smaller than that of a harmonized melody tone. Moreover, the present stimuli were embedded within a complex musical context, and were due to their rule-based arrangement within a (complex) sequence reminiscent to linguistic phrases (Koelsch et al., 2000a; Maess et al., 2001).

These features of the present stimuli may also have contributed to the clear left-hemispheric involvement, which contrasts with some previous imaging studies. However, note that previous lesion studies al ready emphasized the importance of the left hemisphere for the processing of music (Liegeois-Chauvel et al., 1998; Peretz, 1990). Moreover, results obtained in the preceding EEG and MEG studies (Koelsch et al., 2000a; Maess et al., 2001; Koelsch et al., 2001) revealed strong involvement of both hemispheres for the processing of harmonically inappropriate chords, although some effects had clear right predominance in the EEG. The bilateral activation observed in the fMRI-data is thus in line with these findings. Additionally, it seems plausible that left-hemisphere resources might have been particularly activated due to the MR-background noise which made the task more difficult than in the EEG and MEG experiments.

With respect to language processing, activation of the right hemisphere (J ust et al., 1996; Shaywitz et al., 1995; Friederici et al., 2000a), or even right hemispheric preponderance has been reported for the processing of language, especially when on-line repair of auditorily presented sentences is required (Meyer et al., 2000b). Moreover, the right-hemispheric predominance of language-processing has particularly been observed for the processing of prosody (Meyer et al., 2000a, 1999), that is of musical features of language (e.g., melody and metre). The combined findings do not support the notion of a strict lateralization of language processing to the left and music processing to the right hemisphere. Instead, they rather suggest a strong interaction of both hemispheres during the processing of both language and music.

A comparison of the present activations with those elicited by auditory oddballs which are not embedded within a musical context reveal that the latter do not activate the network observed in the present study. Even when presented as deviant stimuli in oddballparadigms (in reminiscence to the inappropriate chords of the present study), complex sounds mainly activate areas within, or in the close vicinity of the 
primary auditory cortex (e.g., Alho et al., 1996; Tervaniemi et al., 2000). Frontal activations have also been reported, although not to the extent observed in the present study, and so far only for the frequencyMMN (e.g., Giard et al., 1990; Alain et al., 1998; Opitz et al., 1999). Notably, the effects elicited by modulations cannot reflect a frequency-MMN, because each chord differed from the previous chord, and notes in the top- or base-voice were never repeated in more than two chords (thus, no physical memory-trace needed to elicit a frequency-MMN could be established). Hence, the present activations cannot just be due to the processing of auditory oddballs or to the mere processing of complex sounds. With this respect, it is highly probable that the embeddedness of a deviant acoustic event in a complex, rule-based linguistic or musical context is a prerequisite for the present activations (for studies investigating the neural mechanisms of timbre processing see also Samson and Zatorre, 1993; Auzou et al., 1995; J ones et al., 1998; Toivianinen et al., 1998; Tervaniemi et al., 1997; Platel et al., 1997; Crummer et al., 1994).

Importantly, since the same neuronal network processes musical and linguistic information, the human musical experiences might implicitly train the language network. Thus, the present results provide an explanation why musical elements participate very early in the process of language development in children: it has been hypothesized that music and speech are intimately connected in early life (Trainor and Trehub, 1992; Trehub et al., 2000), that musical elements pave the way to linguistic capacities earlier than phonetic elements (Papoušek, 1996), and that melodic aspects of adult speech to infants represent the infant's earliest associations between sound patterns and meaning (Fernald, 1989), as well as between sound patterns and syntactic structure (Krumhansl and J usczyk, 1990; J usczyk et al., 1992; J usczyk and Krumhansl, 1993). I mprovement of abilities in the language domain by training in the musical domain has also been reported for adults (Chan et al., 1998; Douglas and Willatts, 1994).

Effects of the three different conditions (modulations, clusters, and deviant instruments) were observed in virtually the same brain regions. This seems surprising, since modulations and clusters were harmonically inappropriate (with an appropriate timbre), and deviant instruments had an inappropriate timbre (being harmonically appropriate). However, due to the task, all conditions required the same thorough processing of both harmonic and timbral information: Modulating chords were harmonically less closely related than in-key chords (and thus perceived by listeners as slightly more unexpected compared to the in-key chords), but modulating chords were nevertheless consonant (in contrast to clusters) and played by the piano (in contrast to deviant instruments). Clusters were not consonant, but had the correct timbre. Deviant instruments had an unexpected timbre, but were harmonically correct. That is, in order to press the appropriate button, participants had to analyze thoroughly all 'deviant' acoustic events (modulations, clusters, and deviant instruments) with respect to both their harmonic and timbral information. This analysis might have contributed to the result that all three conditions activated very similar brain regions.

It is important to note, however, that there are also specific differences between the three conditions: First, the three conditions elicited different activation levels (effects were weakest for modulations and strongest for deviant instruments). The different activation levels correspond to the saliency and to the task demands of the three conditions: subjects had to respond to the deviant instruments, to attend the clusters, and to ignore the modulating sequences. J äncke et al. (1999) showed that auditory simuli elicit the strongest response when they are to be detected (as the deviant instruments in the present study), followed stimuli which are to be attended but do not require a response (as the clusters); the weakest activation is elicited by stimuli which are to be ignored (as the modulations; for similar findings see, e.g., Roland, 1982; Hsiao \& al., 1993). Correspondingly, dueto the task demands the head of the caudate nucleus was possibly activated only during the processing of deviant instruments and dusters: the head of the caudate nucleus (in parallel with the thalamus) has been reported to be activated when task-relevant auditory events occur within a stream of complex auditory information (e.g., sentences; cf. Meyer et al., 2000b).

Clusters were also more salient than modulations (as were, presumably, the deviant instruments). This difference in saliency might also have contributed to a stronger activation of clusters and deviant instruments compared to the modulations. To what extent salience on the one hand, and task-rel evance on the other, modified the strength of activation in the present experiment remains to be specified.

Second, only effects of deviant instruments were lateralized. This finding is in accordance with a substantial amount of literature which reports a right hemisphere dominance for the processing of timbre information (Milner, 1962; Mazzucchi et al., 1982; Samson and Zatorre, 1993; Auzou et al., 1995; J ones et al., 1998). The right predominance for the deviant instruments compared to the clusters is al so reflected in the EEG data of a previous experiment (Koelsch et al., 2000a) in which both clusters and deviant instruments elicited an early ERP-wave which was lateralized to the right, but in which a later ERP-wave was rightlateralized only when elicited by deviant instruments. Thus, the present data support the notion that the right hemisphere plays a dominant role for the processing of timbral information. 
Third, the posterior third of the STS in the right hemisphere and the most posterior part of the STG were only activated by clusters and deviant instruments. The posterior region of the STG (along with the planum temporale, which was in the present study also activated by the modulations) including the upper bank of the STS have been reported to be involved in phoneme identification (Pöppel, 1996). In particular, regions along the banks of the STS have been shown to be involved in prelexical processing (Mummery et al., 1999) and perception of acoustic-phonological features of speech (Binder et al., 1994; Pöppel, 1996). One might draw a parallel between the interval structure of a chord and the phonological structure of a word. Given this similarity between phonemes and intervals, the processing of interval relations (i.e., the illegal interval structure of clusters) might have activated these re gions. The deviant instruments had no illegal interval structure, but the interval structure of these chords had nevertheless to be processed in order to make the correct response. The interval structure of the modulations was legal and matched with the preceding chords (the modulating chords differed from the chords of the standard sequences only with respect to their tonal key). Thus, the posterior STG and the STS was possibly not activated by this condition.

The same reasoning might apply to the finding that the superior part of the pars opercularis (BA 44/6) in the left hemisphere was only activated by the deviant instruments and the clusters, since this region has been reported to be involved in the processing of relations between phonemes, phonological sequences, and phonemic fluency (Friederici, 1998; Pöppel, 1996; Troyer et al., 1998). It remains unclear, however, why this structure was nevertheless activated in the right hemisphere by the modulations.

\section{CONCLUSION}

In essence, the present results indicate that the processing of music relies on a cortical network that comprises inferior fronto-lateral and anterior as well as posterior temporal lobe structures in both hemispheres. This finding has several implications. First, because this network appears to be very similar to the network known to support auditory language processing, results suggest that sequential auditory information is processed by brain structures which are less domain-specific than previously believed. With this respect, the present data do not support a strict dichotomy between auditory language and music processing, but rather suggest considerable overlap, particularly when suprasegmental aspects of sequence processing are in focus. Second, the present results provide functional-neuroanatomical support for the notion that musical elements of speech play a crucial role for the acquisition of language (Trainor and Trehub, 1992;
Trehub et al., 2000; Papoušek, 1996; Fernald, 1989; J usczyk and Krumhansl, 1993), probably giving rise to new didactic and therapeutic implications. Third, results support the notion that music has (like language) a syntactic and a semantic dimension. Fourth, participants of the present study were nonmusicians. Nevertheless, distinct activations were elicited by the modulating sequences, which could only be differentiated from in-key sequences by the application of (implicit) knowledge about music-theoretical principles of harmonic relatedness. Thus, the present results also indicate surprisingly sensitive musical responses in the brains of nonmusicians.

\section{REFERENCES}

Aguirre, G., Zarahn, E., and d'Esposito, M. 1997. Empirical analyses of BOLD fMRI statistics. II. Spatially smoothed data collected under null-hypothesis and experimental conditions. Neurol mage 5: 199-212.

Alain, C., Woods, D. L., and Knight, R. T. 1998. A distributed cortical network for auditory sensory memory in humans. Brain Res. 812: 23-37.

Alho, K., Tervaniemi, M., Huotilainen, M., Lavikainen, J ., Tiitinen, H., I Imoniemi, R., Knuutila, J ., and Näätänen, R. 1996. Processing of complex sounds in the human auditory cortex as revealed by magnetic brain responses. PsPh 33(4): 369-375.

Auzou, P., Eustache, F., Etevenon, P., Platel, H., Rioux, P., Lambert, J., Lechevalier, B., Zarifian, E., and Baron, J . 1995. Topographic EEG activations during timbre and pitch discrimination tasks using musical sounds. Neuropsychologia 33(1): 25-37.

Bellin, P., Zatorre, R., Lafaille, P., Ahad, P., and Pike, B. 2000. Voice-selective areas in human auditory cortex. Nature 403: 309312.

Bharucha, J ., and Krumhansl, C. 1983. The representation of harmonic structure in music: Hierarchies of stability as a function of context. Cognition 13: 63-102.

Bharucha, J., and Stoeckig, K. 1986. Reaction time and musical expectancy: Priming of chords. J. Exp. Psychol. Hum. Percept. Perform. 12: 403-410.

Bigand, E., Madurell, F., Tillmann, B., and Pineau, M. 1999. Effect of Global Structure and Temporal Organization on Chord Processing. J . Exp. Psychol. Hum. Percept. Perform. 25(1): 184-197.

Bigand, E., and Pineau, M. 1997. Global context effects on musical expectancy. Percept. Psychophys. 59(7): 1098-1107.

Binder, J ., Rao, S., Hammeke, T., Yetkin, F., J esmanowicz, A., Bandettini, P., Wong, E., Estkowski, L., Goldstein, M., Haughton, V., and Hyde, J . 1994. Functional magnetic-resonance-imaging of human auditory-cortex. Ann. Neurol. 35: 662- 672.

Blood, A., Zatorre, R., Bermudez, P., and Evans, A. 1999. Emotional responses to pleasant and unpleasant music correlate with activity in paralimbic brain regions. Nature Neurosci. 2(4): 382-387.

Bosch, V. 2000. Statistical analysis of multi-subject fmri data: The assessment of focal activations. J . Magn. Reson. I mag. 11: 61- 64.

Chan, A., Ho, Y., and Cheung, M. 1998. Music training improves verbal memory. Nature 396: 128.

Crummer, G., Walton, J . P., Wayman, J . W., and Hantz, E. C. 1994. Neural processing of musical timbre by musicians, nonmusicians, and musicians possessing absolute pitch. J . Acoust. Soc. Am. 95(5): 2720-2727.

Dapretto, M., and Bookheimer, S. 1999. Form and content: dissociating syntax and semantics in sentence comprehension. Neuron 24: 427- 432 . 
Douglas, S., and Willatts, P. 1994. The relationship between musical ability and literacy skills. J. Res. Read. 17: 99-107.

Fernald, A. 1989. Intonation and communicative intent in mothers' speech to infants: Is the melody the message? Child Dev. 60: 1497-1510.

Friederici, A. D. (Ed.) 1998. Language Comprehension: A Biological Perspective. Springer.

Friederici, A. D., Meyer, M., and v.Cramon, D. Y. 2000a. Auditory language comprehension: An event-related fMRI study on the processing of syntactic and lexical information. Brain Lang. 74: 289300.

Friederici, A. D., Pfeifer, E., and Hahne, A. 1993. Event-related brain potentials during natural speech processing: Effects of semantic, morphological and syntactic violations. Cogn. Brain Res. 1: 183192.

Friederici, A. D., Wang, Y., Herrmann, C., Maess, B., and Oertel, U. 2000b. Localisation of early syntactic processes in frontal and temporal cortical areas: A MEG study. Hum. Brain Mapp. 11: 1-11.

Friston, K. 1994. Statistical parametric maps in functional imaging: A general linear approach. Hum. Brain Mapp. 2: 189-210.

Giard, M., Perrin, F., and Pernier, J . 1990. Brain generators implicated in processing of auditory stimulus deviance. A topographic ERP study. Psychophysiology 27: 627-640.

Grossman, M., Shapiro, B., and Gardner, H. 1981. Dissociable musical processing strategies after localized brain damage. Neuropsychologia 19: 425- 433.

Hahne, A., and Friederici, A. D. 1999. Electrophysiological evidence for two steps in syntactic analysis: Early automatic and late controlled processes. J . Cogn. Neurosci. 11(2): 194-205.

Hindemith, P. 1940. Unterweisung im Tonsatz, 1. Theoretischer Tél. Schott, Mainz.

Holmes, A. P., and Friston, K. J . 1998. Generalisability, random effects \& population inference. Neurol mage 7: 754.

Hsiao, S., O'Shaughnessy, D., and J ohnson, K. 1993. Effects of Selective Attention on Spatial Form Processing in Monkey Primary and Secondary Somatosensory Cortex. J. Neurophysiol. 70(1): 444- 447.

J äncke, L., Mirzazade, S., and Shah, N. 1999. Attantion modulates activity in the primary and the secondary auditory cortex: A functional magnetic resonance imaging study in human subjects. Neurosci. Lett. 266: 125-128.

J ones, S., Longe, O., and Vaz Pato, M. 1998. Auditory evoked potentials to abrupt pitch and timbre change of complex tones: electrophysiological evidence of "streaming"? Electroencephalog. Clin. Neurophysiol. 108: 131-142.

J usczyk, P., Hirsh-Pasek, K., Kemler-Nelson, D., Kennedy, L., Woodward, A., and Piwoz, J . 1992. Perception of acoustic correlates of major phrasal units by young infants. Cogn. Psychol. 24: 252-293.

J usczyk, P. W., and Krumhansl, C. L. 1993. Pitch and rhythmic patterns affecting infants' sensitivity to musical phrase structure. J . Exp. Psychol. Human Perception \& Performance 19(3): 627- 640.

J ust, M., Carpenter, P., Keller, T., Eddy, W., and Thulborn, K. 1996. Brain activation modulated by sentence comprehension. Science 274: 114-116.

Koelsch, S., Gunter, T., Friederici, A. D., and Schröger, E. 2000a. Brain indices of music processing: "N on-musicians" are musical. J . Cogn. Neurosci. 12(3): 520-541.

Koelsch, S., Gunter, T., Schröger, E., Tervaniemi, M., Sammler, D., and Friederici, A. D. 2001. Differentiating ERAN and MMN: An ERP-study. NeuroReport 12(7): 1385-1389.

Koelsch, S., Maess, B., and Friederici, A. D. 2000b. Musical syntax is processed in the area of Broca: an MEG study. Neuroimage 11(5): 56.
Koelsch, S., Schmidt, B., and Kansok, J . Influences of musical expertise on the ERAN: An ERP-study. Psychophysiology, in press.

Koelsch, S., Schröger, E., and Gunter, T. 2002. Music Matters: Preattentive musicality of the human brain. Psychophysiology 39: $1-11$.

Krumhansl, C., and Kessler, E. 1982. Tracing the dynamic changes in perceived tonal organization in a spatial representation of musical keys. Psychol. Rev. 89(4): 334-368.

Krumhansl, C. L., and J usczyk, P. W. 1990. Infants' perception of phrase structure in music. Psychological Science 1(1): 70-73.

Liegeois-Chauvel, C., Peretz, L., Babaie, M., Laguitton, V., and Chauvel, P. 1998. Contribution of different cortical areas in the temporal lobes to music processing. Brain 121(10): 1853-1867.

Lohmann, G., Müller, K., Bosch, V., Mentzel, H., Hessler, S., Chen, L., and V.Cramon, D. Y. in press. Lipsia-A new software system for the evaluation of functional magnet resonance images of the human brain. Comput. Med. Imag. Graphics. See also at http:// www.cns.mpg.de/lipsia.

Maess, B., Koelsch, S., Gunter, T., and Friederici, A. D. 2001. “Musical Syntax" is processed in the Broca's area: An MEG-study. Nature Neurosci. 4(5): 540-545.

Mayer, J ., Dogil, G., Ackermann, H., Erb, M., Grodd, W., Haider, H., Riecker, A., and Wildgruber, D. 1999. Prosody in speech production. Neurol mage 9: S1065.

Mazoyer, B., Tzourio, N., Frak, V., Syrota, A., Murayama, N., Levrier, O., Salamon, G., Dehaene, S., Cohen, L., and Mehler, J . 1993. The cortical representation of speech. J . Cogn. Neurosci. 5(4): 467-479.

Mazzucchi, A., Marchini, C., Budai, R., and Parma, M. 1982. A case of receptive amusia with prominent timbre perception defect. J . Neurol. Neurosurg. Psychiatry 45: 644-647.

Meyer, M., Alter, K., Friederici, A. D., and v.Cramon, D. Y. 2000a. Different hemodynamic responses to sentence-level syntactic and prosodic processing. Neurol mage 11(5): S281.

Meyer, M., Friederici, A. D., and v.Cramon, D. Y. 2000b. Neurocognition of auditory sentence comprehension: Event related fMRI reveals sensitivity to syntactic violations and task demands. Cogn. Brain Res. 9: 19-33.

Milner, B. 1962. Laterality effects in audition. In Interhemisphere Relations and Cerebral Dominance(V. Mountcastle, Ed.), pp. 177194. J. Hopkins Press, Baltimore.

Mummery, C., Ashburner, J ., Scott, S., and Wise, R. 1999. Functional neuroimaging of speech perception in six normal and two aphasic subjects. J . Acoust. Soc. Am. 106: 449- 457.

Opitz, B., Mecklinger, A., von Cramon, D. Y., and Kruggel, F. 1999. Combining electrophysiological and hemodynamic measures of the auditory oddball. Psychophysiology 36(1): 142-147.

Papoušek, H. 1996. Musicality in infancy research. In Musical Be ginnings (J . Sloboda and I. Deliege, Eds.) Oxford Univ. Press, Oxford.

Patel, A. D., Gibson, E., Ratner, J ., Besson, M., and Holcomb, P. 1998. Processing syntactic relations in language and music: An event-related potential study. J . Cogn. Neurosci. 10(6): 717-733.

Peretz, I. 1990. Processing of local and global musical information by unilateral brain-damaged patients. Brain 113: 1185- 1205.

Peretz, I., Kolinsky, R., Tramo, M., and Labrecque, R. 1994. Functional dissociations following bilateral lesions of auditory cortex. Brain 117(6): 1283-1301.

Platel, H., Price, C., Baron, J ., Wise, R., Lambert, J ., Frackowiak, R., Lechevalier, B., and Eustache, F. 1997. The structural components of music perception, A functional anatomical study. Brain 120: 229-243.

Pöppel, D. 1996. A Critical Review of PET Studies of Phonological Processing. Brain Lang. 55: 317-351. 
Raffmann, D. 1993. Language, Music, and Mind. MIT Press, Cambridge.

Roland, P. 1982. Cortical regulation of selective attention in man. A regional cerebral blood flow study. J . Neurophysiol. 48(5): 10591077.

Samson, S., and Zatorre, R. 1988. Melodic and harmonic discrimination following unilateral cerebral excision. Brain Cogn. 7: 348-360.

Samson, S., and Zatorre, R. 1993. Contribution of the right temporal lobe to musical timbre discrimination. Neuropsychologia 32(2): 231-240.

Schlosser, M., Aoyagi, N., Fulbright, R., Gore, J ., and McCarthy, G. 1998. Functional MRI studies of auditory comprehension. Hum. Brain Mapp. 6(1): 1-13.

Schönberg, A. 1969. Structural Functions of Harmony. Norton, rev. edition, New York.

Shapiro, B., Grossman, M., and Gardner, H. 1981. Selective processing deficits in brain damaged populations. Neuropsychologia 19: 161-169.

Shaywitz, B., Shaywitz, S., Pugh, K., Constable, R., Skudlarski, P., Fulbright, R., Bronen, R., Fletcher, J ., Shankweiler, D., Katz, L., and Gore, J . 1995. Sex differences in the functional organization of the brain for language. Nature 373: 607-609.

Swain, J . 1997. Musical Languages. Norton, UK.

Talairach, J ., and Tournoux, P. 1988. Co-Planar Stereotaxic Atlas of the Human Brain. 3-Dimensional Proportional System: An Approach to Cerebral I maging. Thieme, Stuttgart.

Tervaniemi, M., Medvedev, S., Alho, K., Pakhomov, S., Roudas, M., v.Zuijen, T., and Näätänen, R. 2000. Lateralized automatic auditory processing of phonetic versus musical information: A PET study. Hum. Brain Mapp. 10(2): 74-79.

Tervaniemi, M., Winkler, I., and Näatänen, R. 1997. Pre-attentive categorization of sounds by timbre as revealed by event-related potentials. Neuroreport 8(11): 2571-4.

Tillmann, B., Bharucha, J ., and Bigand, E. 2000. I mplicit learning of tonality: A self-organized approach. Psychol. Rev. 107(4): 885-913.
Tillmann, B., Bigand, E., and Pineau, M. 1998. Effects of global and local contexts on harmonic expectancy. Music Percept. 16(1): 99117.

Toiviainen, P., Tervaniemi, M., Louhivuori, J ., Saher, M., Huotilainen, M., and Näätänen, R. 1998. Timbre similarity: Convergence of neural, behavioral, and computational approaches. Music Percept. 16(2): 223-241.

Trainor, L., and Trehub, S. 1992. A comparison of infants' and adults' sensitivity to western musical structure. J EPP 18(2): 394-402.

Trehub, S., Schellenberg, G., and Hill, D. 2000. The origins of music: Perception and cognition: A developmental perspective. In The Origins of Music (N. L. Wallin, B. Merker, and S. Brown, Eds.) MIT Press, Cambridge, MA.

Troyer, A., Moscovitch, M., Winocur, G., Alexander, M., and Stuss, D. 1998. Clustering and switching on verbal fluency: The effects of focal frontal- and temporal-lobe lesions. Neuropsychologia 36(6): 499-504.

Worsley, K., and Friston, K. 1995. Analysis of fMRI time-series revisited-Again. Neurol mage 2: 359-365.

Zarahn, E., Aguirre, G., and D'Esposito, M. 1997. Empirical analyses of BOLD fMRI statistics. Neurol mage 5: 179-197.

Zatorre, R. 1984. Musical perception and cerebral function: a critical review. Music Percep. 2(2): 196-221.

Zatorre, R. 1985. Discrimination and recognition of tonal melodies after unilateral cerebral excisions. Neuropsychol ogia 23(1): 31- 41.

Zatorre, R., Evans, A., and Meyer, E. 1994. Neural mechanisms underlying melodic perception and memory for pitch. J . Neurosci. 14(4): 1908-1919.

Zatorre, R., Evans, A., Meyer, E., and Gjedde, A. 1992. Lateralization of phonetic and pitch discrimination in speech processing. Science 256: 846-849.

Zatorre, R., and Samson, S. 1991. Role of the right temporal neocortex in retention of pitch in auditory short-term memory. Brain 114: 2403-2417. 